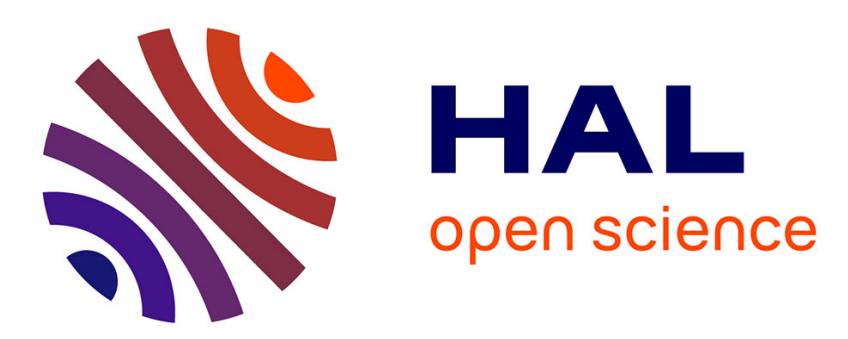

\title{
A Signal Processing Method for Artefact Rejection in Transcranial Doppler Signals used for Micro-embolus detection
}

Maroun Geryes, Sébastien Ménigot, Jamal Charara, Ahmad Skaiky, Ali Mcheick, Jean Marc Girault

\section{To cite this version:}

Maroun Geryes, Sébastien Ménigot, Jamal Charara, Ahmad Skaiky, Ali Mcheick, et al.. A Signal Processing Method for Artefact Rejection in Transcranial Doppler Signals used for Micro-embolus detection. ICABME 2019, Oct 2019, Tripoli, Lebanon. 10.1109/ICABME47164.2019.8940150 . hal02346758

\section{HAL Id: hal-02346758 \\ https://hal.science/hal-02346758}

Submitted on 5 Nov 2019

HAL is a multi-disciplinary open access archive for the deposit and dissemination of scientific research documents, whether they are published or not. The documents may come from teaching and research institutions in France or abroad, or from public or private research centers.
L'archive ouverte pluridisciplinaire HAL, est destinée au dépôt et à la diffusion de documents scientifiques de niveau recherche, publiés ou non, émanant des établissements d'enseignement et de recherche français ou étrangers, des laboratoires publics ou privés. 


\title{
A Signal Processing Method for Artefact Rejection in Transcranial Doppler Signals used for Micro-embolus detection
}

\author{
Maroun Geryes*, Sébastien Ménigot ${ }^{\dagger \ddagger}$, Jamal Charara*, \\ Ahmad Skaiky ${ }^{\S}$, and Ali Mcheick ${ }^{\natural}$, Jean-Marc Girault ${ }^{\dagger \ddagger}$, \\ * Department of Physics and Electronics, Faculty of Sciences I, Lebanese University \\ $\dagger$ LAUM, UMR-CNRS 6613, Université du Maine, Le Mans, France \\ $\ddagger$ ESEO Group, Angers, France \\ $\S$ Arts, Sciences and Technology University in Lebanon \\ ${ }^{\top}$ Faculty of Agriculture, Lebanese University
}

\begin{abstract}
Atefacts are High Intensity Transient Signals that appear in the Doppler signal due to probe tapping, probe displacement, patient movement and other external factors during TCD recording. In Holter TCD, the number of artefacts are further increased due to the servo-controlled probe positioning and long recording time. Current artefact rejection methods must be adapted to the new holter devices. Therefore, in this paper we present a complete procedure for eliminating two types of artefacts that occur during Holter TCD. The latter two types are bidirectional areftact, occurring in the energy of both positive and negative frequencies, and unidirectional artefacts occuring only in the energy of the negative frequencies. From a dataset of 25 signals, 218 artefact signatures were identified; $95 \%$ of which are bidirectional and 5\% unidirectional. As a final result, $98 \%$
\end{abstract} of the artefacts where successfully removed.

Keywords- Holter TCD; artefact rejection; bidirectional artefacts; unidirectional artefacts

\section{INTRODUCTION}

CerebroVascular Accidents (CVAs) are the second cause of mortality worldwide. CVAs represent a major concern and are considered as a public health issue. Early diagnosis and treatment of CVAs would help in avoiding its occurrence. An effective widely used CVA diagnosis solution is the Transcranial Doppler (TCD) system [1]. TCD commercial systems include lengthy probe positioning procedures, are unreliable in detecting microemboli, and provide short examination durations. The Holter TCDs have offered solutions for the latter restrictions such as servo-controlled positioning of the ultrasound probe and prolonged patient monitoring [2].

Sudden intensity increases in the Holter Transcranial Doppler (TCD) signal are majorly interpreted as signatures resulting from cerebral emboli. CVAs resulting from emboli represent a major death threat and thus the early detection of the smallest micro-emboli is an important issue.

Generally, the high intensity transient increases (HITS) appearing in the TCD signal are termed as embolic signals. However, the latter terminology would be misleading due to the presence of signals that are similar to embolic signals and also have the characteristics of being HITS. These are artefact signals. Artefacts can be misinterpreted as true microembolic signals and thus can greatly mislead the embolus detection system. Indeed, misinterpreting artefacts as embolic detections has the potential to mislead researchers and also readers into thinking the embolus detection may be more clinically valuable than it actually is.

Artefacts are majorly caused by Doppler probe tapping or displacement. The servo-controlled probe positioning in Holter TCD results in a great increase in the number of signal artefacts. Moreover, due to the long recording time, the patient is more likely to cause artefacts due to movements and normal actions that are difficult to control.

Therefore, the development of new microemboli detectors should be completed with artefact rejection in the TCD signals. Artefact rejection becomes a mandatory step engaged in the whole detection process. At our end, we have developed several embolus detection systems. In this paper, we propose an artefact rejection procedure that ensures eliminating artefacts present in our database signals. This step will help in achieving an artefact-free clean database signals and thus in providing more reliable embolus detection reports.

A widely known fundamental method to separate artefacts from emboli is the dual-gate or multi-gate TCD [3] [4], [5] . The technique is based on tracking and tracing the movement of the embolus at 2 separate depths in the insonated artery. Therefore, more than one Doppler sample volume is used.

However when a single-gated system is to be used, other artefact features should be considered to perform rejection. The latter case applies when the Holter system is used. This system allows only single gated emission. Therefore, in this paper we represent Doppler single-gated based artefact rejection procedures.

\section{Holter Monitoring And Data Collection}

The Transcranial Holter is an advanced generation of the conventional Transcranial Doppler system. It has been implemented by Atys Medical (Soucieu en Jarrest, France) to offer improved options and characteristics. These include prolonged patient monitoring up to more than 5 hours, patient monitoring 
under naturalistic conditions were the patient is no longer attached to a TCD, and the use of a robot probe that helps find the best TCD signal and tracks it automatically during the whole recording. The Holter recordings were carried out at the hospital of Lille (Centre Hospitalier Régional Universitaire de Lille, 2 Avenue Oscar Lambret, 59000 Lille, France). Patients with carotid stenosis were chosen for monitoring. Written approval was obtained from all patients under monitoring. The written consent forms were investigated and authorized by the hospital of Lille. The Holter transducer emits an ultrasound wave with a $1.5 \mathrm{MHz}$ frequency to the middle cerebral artery of the patients. A pulse repetition frequency (PRF) of $6.4 \mathrm{kHz}$ is used with an ultrasound power of $50 \mathrm{~mW} / \mathrm{cm}^{-2}$.

After the clinical procedure, the Holter recordings are converted into audio-wave files (quantification over 16 bits and sampling frequency of $4 \mathrm{kHz}$ ). In the following postprocessing phase, the audio files are analyzed and manipulated with our developed algorithms in the numerical calculation software Matlab (Mathworks, Natick, MA, USA).

\section{METHODS}

\section{A. Pre-considerations}

When using single-gated TCDs, the leading method for artefact rejection depends on a strong hypothesis stating that in the Doppler blood flow velocity spectrum artefacts are bidirectional while emboli are unidirectional. This method has been extensively stated by [6], [7], [8], [9] and [10], although the latter studies did not present or prove the artefact rejection phase. This means that artefacts exhibit velocity components both in the forward and reverse flows while emboli exhibit velocity components in the forward flow only. The forward flow denotes the channel that contains the main flow of blood while in the reverse no blood flow is present (noise signal).

The Doppler signal is a complex signal where the signal of the positive frequencies can be different from the signal of the negative frequencies. Nevertheless, for strong over-intensities (overdriving electronics) the content of positive and negative frequency components can be very similar, or even the same. Note that these issues create artefacts mainly considered as bidirectional, because either they are really bidirectional or they are detected as bidirectional. However, this hypothesis is valid, only if there is no energy in the negative frequencies. Therefore, in this method, we assume that the clinician sets the probe to obtain the blood flow in the positive frequencies only. This implies that there is no blood flow information in the negative frequencies. Thus, the signatures in the negative frequencies are only related to artefacts, while the signatures in the positive frequencies are related to the blood flow and artefacts.

Artefacts are not necessarily bidirectional, but the corresponding signals usually overdrive the electronics and result in presence of signal in both parts of the spectrogram. A previous study [11] showed that artefact signals, even if rarely occurring, could be exclusively unidirectional in the forward flow (positive frequency). In one case of artefacts caused by probe displacement, it was shown [11] that when this probe
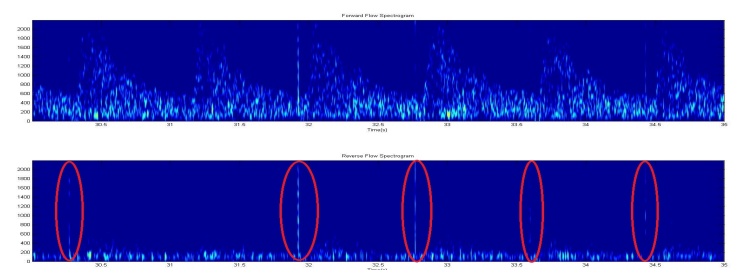

Figure 1: A representation of the forward flow and reverse flow spectrograms of a 5 second signal segment containing 5 artefacts. Note that in many cases artefacts show a straight line running through all the vertical spectrum.

displacement happens in the same forward sense as the blood flow, tissue and wall motion artefacts will always show in the forward Doppler spectrum.

Spectrograms were visualized in order to constitute the gold standard of the artefact detection. Artefact signatures were manually identified on the basis of visual inspection of the spectrogram by three blinded observers of our laboratory. These artefact signatures could be detected equally by both experts and non-experts, since the experience level of the experts is not considered as a critical factor. Thus the time positions of artefacts found by all observers were recorded.

For the next sections we describe the rejection of bidirectional and unidirectional artefacts separately. The energy of the negative frequencies is monitored, and a threshold is applied to detect if the artefacts are unidirectional or not.

\section{B. Rejecting Bidirectional Artefacts}

Since the majority of artefacts have a bidirectional behavior, we proceed with the rejection of this type of artefacts. For a better understanding of the procedure, we present in Figure 1 the forward and reverse spectrograms of a signal segment containing 5 artefact signatures. The spectrogram can be obtained by calculating the Short Time Fourier Transform (STFT). The STFT frequency estimator with a sliding window can be formally written as:

$$
S(t, f)=\left|\int x(\tau) \cdot w^{*}(t-\tau) \cdot \exp ^{-2 \pi j f t} \mathrm{~d} \tau\right|^{2},
$$

The calculated energies from these spectrograms are presented in Figure 2. The instantaneous energy at a fixed time $t$ can be obtained from the frequency estimators in equation 1 by:

$$
E(t)=\int S(t, f) \mathrm{d} f .
$$

As can be seen from the two figures, the artefact signals can be clearly depicted in the reverse flow spectrogram and in the calculated reverse energy signal. Note that these artefacts are of electronic nature since they appear periodically.

The first step in this artefact rejection procedure is to identify and detect the artefacts in the reverse flow energy signal. The artefacts in this blood flow-free energy signal are always associated with higher peaks than the background noise. Thus, they can be detected by a peak detection algorithm 


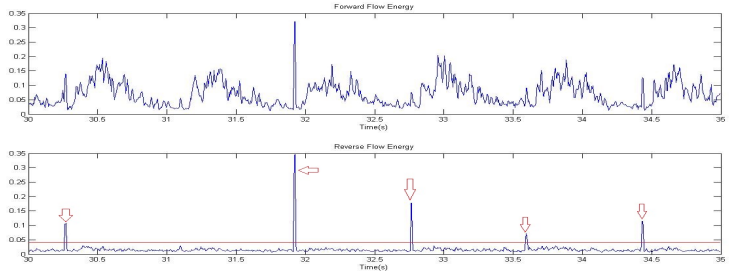

Figure 2: A representation of the forward flow and reverse flow energy signals calculated from the spectrograms in Figure 1 with electrical artefact present in the positive and negative frequency.

or by choosing a constant threshold whose value is greater than the maximum background level as shown in Figure 2. For instance, a threshold superior to $95 \%$ percentiles could be used. Once the artefacts in the reverse flow are detected, the next step is to cancel these artefacts at their same positions in the forward flow. In fact, here we eliminate and replace the corrupted part by the artifact, through interpolating on both sides the area where the artifact has been identified. For clarification, we note that the artefact rejection is achieved on the forward flow signal since it is the signal where embolus detection is performed. Rejecting artefacts in the reverse flow is of no value.

Automatic Holter probe displacement Artefacts: Due to the automatic nature of the robotized Holter probe, this probe automatically displaces from one position to another at some instants during the recording time. During such rapid short movements, the signal recording is halted until the probe displaces and restarts again. After this restart of the probe insonification, an artefact associated to the probe displacement is directly recorded within an interval of 100 to 200 milliseconds. These occuring artefacts are eliminated by the method proposed in section III-B. An example of this type of artefacts is given in Figure 3 and Figure 4. We indicate in Figure 4, for the signature at $41.5 \mathrm{~s}$, that when the probe moves the signal will be zero during a duration of $100 \mathrm{~ms}$.

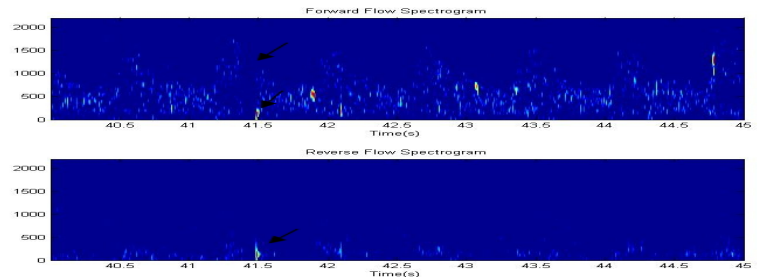

Figure 3: A representation of artefacts resulting from the automatic Holter probe displacements.

\section{Rejecting Unidirectional Artefacts}

While the bidirectional artefacts in the database were identified by using the reverse flow, the unidirectional artefacts were identified based on their frequency component in the forward flow spectrogram. The forward flow was used since

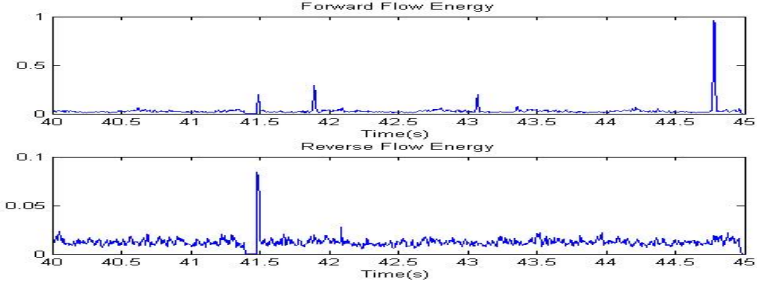

Figure 4: A representation of the signature of an artefact resulting from probe displacement in the reverse and forward flow energy signals. Both flow signals are halted when the probe is in displacement. This artefact is rejected using the method of section III-B. Note that over the position of the artefact a line of zeros is generated throughout the artefact.

the study of [11] had demonstrated that artefacts could be unidirectional particularly in the forward flow. An example of a unidirectional artefact is presented in the spectrogram in Figure 5. A representation of the associated forward flow and reverse flow energy signals is presented in Figure 6. The identification of the unidirectional artefacts in our database was primarly based on frequency since it has been widely mentioned that artefacts have lower frequencies than emboli [12], [11], [13]. Therefore, we determined the embolic and artefact frequency range.

After a study on the frequency content was done, the artefact frequency ranges from $125 \mathrm{~Hz}$ to $550 \mathrm{~Hz}$ (the embolic frequency was found to be between $631 \mathrm{~Hz}$ and $2005 \mathrm{~Hz}$ ). Then a search was initialized in the forward spectrogram for signatures with frequencies between $125 \mathrm{~Hz}$ to $550 \mathrm{~Hz}$ that would correspond to artefacts. If no corresponding signature is found in the reverse flow, then the forward signature is recorded as a unidirectional artefact. Moreover, in order to perfectly confirm that these signatures are artefacts, we searched for artefact-embolus discriminative parameters based on the energy signal rather than on the spectrogram.

Two discriminative parameters were found, the rise rates (RR) and fall rates (FR). These two parameters could assure the signature found in the forward flow spectrogram is an artefact. The results of the rise rates and false rates for both embolic and artefact signals are presented in Figure 7. Embolic signal rise rates range between 0.015 and $0.05 \mathrm{~s}$ while the fall rates range between 0.02 and $0.03 \mathrm{~s}$. Artefact signal rise rates range between 0.002 and $0.01 s$ while the fall rates range between 0.002 and $0.015 \mathrm{~s}$. At the end, 11 unidirectional artefacts were depicted and confirmed and then rejected by the same method in section III-B by setting them to the value of the smoothed version of the forward signal.

To sum up the method, a pseudo-code is available in the appendix.

\section{RESUlts}

In order to discover the directionality properties of the artefacts we have in our database, we tested 218 artefacts using spectrogram visualization of the forward and reverse flows. 

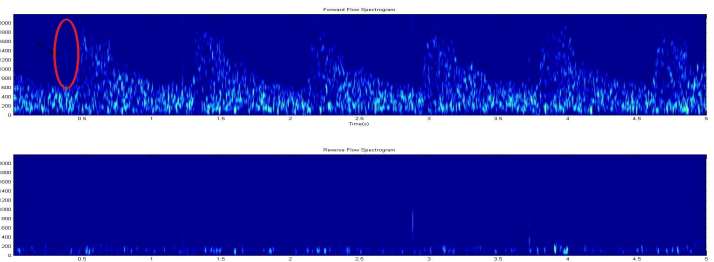

Figure 5: A spectrogram representation showing a unidirectional forward artefact

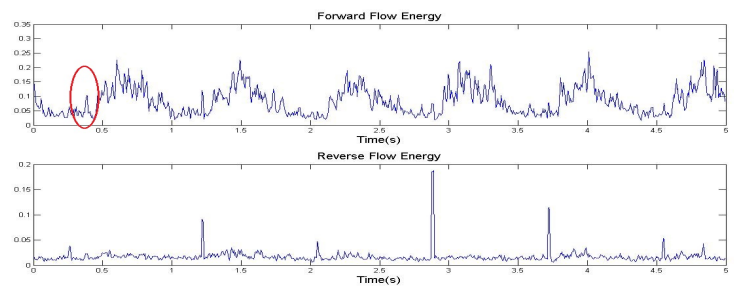

Figure 6: A representation of the forward flow and reverse flow energy signals calculated from the spectrograms in Figure 5

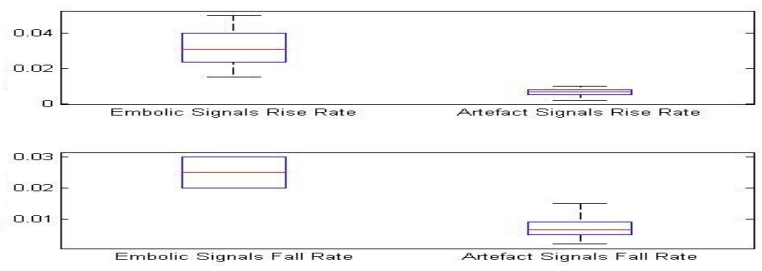

Figure 7: A box plot showing the rise rates and fall rates of embolic and artefact signals. Embolic signal rise rates range between 0.015 and $0.05 \mathrm{~s}$ while the fall rates range between 0.02 and $0.03 \mathrm{~s}$. Artefact signal rise rates range between 0.002 and $0.01 s$ while the fall rates range between 0.002 and $0.015 s$.

The results showed that $95 \%$ of the artefacts are bidirectional and $5 \%$ are unidirectional.

Out of 218 artefact signatures, from 25 signals detected in our database, 207 are bidirectional artefacts (95\%).

By applying the bidirectional procedure for removing artefacts we succeeded in removing 202 out of these 207 artefacts. The bidirectional rejec, tion rate is $97.5 \%$.

Moreover, 11 artefacts, 5\% of the total artefacts, were identified as unidirectional forward artefacts and were rejected. Therefore, the unidirectional artefact rejection rate is $100 \%$.

Therefore in total, out of the 218 artefacts, 213 were successfully removed, with a total rejection rate of $98 \%$ approximately.

\section{Discussion AND CONCLUSION}

High intensity transient signals produced due to artefacts generated during the TCD recording could highly affect the performance and reliability of any complete embolus detection process. For this reason, we have built an artefact rejection system applied before initialization of the embolus detection procedures in order to ensure that signals are artefact-free clean signals. The artefact rejection phase provides more reliability and precision to any embolus detection systems proposed.

We have covered the rejection and elimination of different bidirectional and unidirectional artefacts we might encounter in the database. The methods are founded on the fact that the Holter system is single-gated. Thus we prove that it is possible to robustly reject artefacts without necessarily using standard rejection procedures based on dual-gated or multi-gated TCDs.

The method achieved around $98 \%$ rejection rate. $2.5 \%$ of bidirectional artefacts were not rejected by the system due to their extremely low energy in the reverse flow. This result would help build more reliable embolus detection systems and detection reports and would help reduce embolus false alarm rate.

\section{REFERENCES}

[1] V. Vuković-Cvetković, "Microembolus detection by transcranial doppler sonography: review of the literature," Stroke research and treatment, vol. 2012, 2011.

[2] A. D. Mackinnon, R. Aaslid, and H. S. Markus, "Long-term ambulatory monitoring for cerebral emboli using transcranial doppler ultrasound,' Stroke, vol. 35, no. 1, pp. 73-78, 2004.

[3] J. Molloy and H. S. Markus, "Multigated doppler ultrasound in the detection of emboli in a flow model and embolic signals in patients," Stroke, vol. 27, no. 9, pp. 1548-1552, 1996.

[4] D. Evans, "Multigate emboli detection," in Cerebrovascular Ultrasound: Theory, practice and future developments, Ed. eds, Cambridge University Press, Cambridge, 2001, pp. 360- 373., 2001.

[5] R. Brucher and D. Russell, "Automatic online embolus detection and artifact rejection with the first multifrequency transcranial doppler," Stroke, vol. 33, no. 8, pp. 1969-1974, 2002.

[6] E. B. Ringelstein, D. W. Droste, V. L. Babikian, D. H. Evans, D. G. Grosset, M. Kaps, H. S. Markus, D. Russell, M. Siebler et al., "Consensus on microembolus detection by tcd," Stroke, vol. 29, no. 3, pp. 725-729, 1998.

[7] N. Aydin, F. Marvasti, and H. S. Markus, "Embolic doppler ultrasound signal detection using discrete wavelet transform," IEEE Transactions on Information Technology in Biomedicine, vol. 8, no. 2, pp. 182-190, 2004.

[8] Y. Choi, M. Saqqur, T. Asil, A. Jin, E. Stewart, C. Stephenson, M. Ibrahim, J. Roy, J.-M. Boulanger, S. Coutts et al., "A combined power $\mathrm{m}$-mode and single gate transcranial doppler ultrasound microemboli signal criteria for improving emboli detection and reliability," Journal of Neuroimaging, vol. 20, no. 4, pp. 359-367, 2010.

[9] G. Serbes, B. Sakar, N. Aydin, and H. Gulcur, "An emboli detection system based on dual tree complex wavelet transform," in XIII Mediterranean Conference on Medical and Biological Engineering and Computing 2013. Springer, 2014, pp. 819-822.

[10] B. K. Guépié, B. Sciolla, F. Millioz, R. Ramassamy, E. Pinatel, M. Almar, B. Guibert, and P. Delachartre, "Time-frequency blood flow parameters estimation for micro-emboli detection," in 2015 9th International Symposium on Image and Signal Processing and Analysis (ISPA). IEEE, 2015, pp. 66-71.

[11] M. Biard, D. Kouame, J. Girault, and F. Patat, "Discrimination between emboli and artifacts during transcranial doppler," in the fifth World Congress on Ultrasonics Proceedings (WCU 2003), Paris, 2003.

[12] G. Devuyst, G. Darbellay, J.-M. Vesin, V. Kemeny, M. Ritter, D. Droste, C. Molina, J. Serena, R. Sztajzel, P. Ruchat et al., "Automatic classification of hits into artifacts or solid or gaseous emboli by a wavelet representation combined with dual-gate tcd," Stroke, vol. 32, no. 12, pp. 2803-2809, 2001.

[13] G. A. Darbellay, R. Duff, J.-M. Vesin, P.-A. Despland, D. W. Droste, C. Molina, J. Serena, R. Sztajzel, P. Ruchat, T. Karapanayiotides et al., "Solid or gaseous circulating brain emboli: are they separable by transcranial ultrasound?" Journal of cerebral blood flow \& metabolism, vol. 24, no. 8, pp. 860-868, 2004. 